Check for updates

Cite this: RSC Adv., 2017, 7, 55885

Received 20th October 2017

Accepted 20th November 2017

DOI: 10.1039/c7ra11602k

rsc.li/rsc-advances

\section{Enhancement of light-emitting properties by simultaneously patterning and controlling molecular alignment in polyfluorene thin films $\uparrow$}

\author{
X. H. Li, ${ }^{a}$ X. Z. Shen, ${ }^{a}$ X. Gao ${ }^{b}$ and Y. Y. Weng (D) *a
}

Soft nano-imprinting lithography is used to fabricate PFO nanogratings. On the one hand, soft nanoimprinting lithography has been well explored to create nanostructures and induce molecular orientation in conjugated polymer thin films because of its limited cost, high throughput, and small feature size. On the other hand, the pure $\alpha$-conformation PFO nanogratings with highly ordered molecular chains can be achieved with graphoepitaxial alignment without any molecular chain degradation. Hence, herein, we demonstrated that large-area PFO (poly(9,9-dioctylfluorene)) nanogratings could be fabricated by this effective way with high accuracy of patterning and chain alignment controllability. The molecular chain alignment degree can be tuned due to entropic and geometrical reasons; this has been verified by polarized absorption and GIWAXD (grazing incidence wide angle X-ray diffraction). Moreover, both the optical and electrical pumping properties have been characterized. In addition, not only optimized polarizability was observed at the most oriented situation, but also $20 \%$ more light was extracted from this PLED device.

\section{Introduction}

With the development of LEDs, cold cathode fluorescent lamps have been significantly replaced because of their power consumption, heat, brightness, and lifetime. LED has been widely used in laptops, cell phones, and other flat panel displays. Moreover, PLED have dominated almost half the market of the LED on display backlights as they are flat, thin, and flexible. A polarized light source is more preferred for PLED display as a traditional absorbing sheet polarizer is not very efficient. More than $50 \%$ of light would remain unused when unpolarized light is passed through a polarizer to obtain polarized light. ${ }^{\mathbf{1 - 3}}$ Therefore, many approaches, such as rubbing, ${ }^{4}$ stretching, ${ }^{5}$ and friction-transfer, ${ }^{6-8}$ have been developed to induce chain alignment in thin films. An additional polymer layer, such as a rubbed polyimide film 9 or a photo-induced azobenzene film, has also been introduced into these approaches..$^{10,11}$ Most of these assisting thin layers are good insulators, whose thicknesses do not exceed tens of nanometers in the traditional PLED 5-layer structure. Hence, the molecular chain alignment without any

${ }^{a}$ Centre for Soft Condensed Matter Physics and Interdisciplinary Research, College of Physics, Optoelectronics and Energy, Soochow University, Suzhou, 215006, China. E-mail:wengyuyan@suda.edu.cn

${ }^{b}$ Institute of Functional Nano \& Soft Materials (FUNSOM), Collaborative Innovation Centre of Suzhou Nano Science \& Technology, Soochow University, Suzhou 215006, China

† Electronic supplementary information (ESI) available. See DOI: $10.1039 / \mathrm{c} 7 \mathrm{ra} 11602 \mathrm{k}$ assisting layers would be more appreciated. Considering this, a number of studies have been focused on confinement-induced molecular chain alignment during the nanoimprint process. ${ }^{\mathbf{1 2 - 1 4}}$

Because of high luminescence efficiency, relatively high charge carrier mobility, and excellent thermal stability, PFO is a good candidate for a blue-emitting active layer on PLED devices, ${ }^{15-17}$ and the alignment of these molecular chains can result in the emission of polarized blue light. ${ }^{18-20}$ As a rod-like conjugated polymer, the PFO molecular chain arrangement can be affected by graphoepitaxial alignment and nanoconfinement in the cavities of the nanoimprinting templates. ${ }^{13,21,22}$ By efficiently tuning the PFO molecular chain alignment degree in the active layer, polarizability improvement can be realized.

On the other hand, it has been reported that photonic crystals formed on the surface or in the active layer can be used to extract more light from the PLED devices. ${ }^{23-25}$ However, it is difficult to simultaneously introduce photonic crystal structures and induce chain alignment in one active layer of PLED. Herein, we demonstrated that both achievements could be directly realized on nanoembossing PFO thin films. In this study, onestep fabrication of the active layer of PLED has been demonstrated, which can spontaneously realize high emission intensity and polarizability.

\section{Results and discussion}

PFO (poly(9,9-dioctylfluorene)) was purchased from American dye source company and used as received, and the molecular weight of PFO $\left(M_{\mathrm{w}}=68000 \mathrm{~g} \mathrm{~mol}^{-1}, \mathrm{PDI}=4.3\right)$ is supported by 
the American dye source company. The PFO powder was dissolved in toluene, and the PFO thin films were prepared by spincoating. The substrates used were quartz glass and silicon wafer, cleaned in freshly prepared piranha solution $\left(\mathrm{H}_{2} \mathrm{O}_{2}(27 \%)\right.$ and $\mathrm{H}_{2} \mathrm{SO}_{4}$ (98\%) $1: 3$ ). The film thickness depended on both the spin speed and the solution concentration. To control the thickness of the spin-coated film in the range between $25 \mathrm{~nm}$ and $90 \mathrm{~nm}$, solution concentration in the range from $10 \mathrm{mg}$ $\mathrm{ml}^{-1}$ to $20 \mathrm{mg} \mathrm{ml}^{-1}$ was chosen, and spin speeds were between $2000 \mathrm{rpm}$ and $4000 \mathrm{rpm}$. The $\alpha$-conformation thin film achieved by thermal annealing at $180{ }^{\circ} \mathrm{C}$ for 5 minutes was slowly cooled down to $135{ }^{\circ} \mathrm{C}$, kept for 10 minutes at this temperature, and then cooled down to room temperature. These spin-coated films were transferred to a nanoimprinter (Obducat, Eitre 3) and covered with PDMS (polydimethylsiloxane) template replicated from the polymethylmethacrylate (PMMA) template by E-beam lithography. The PDMS templates were pressed against the films under pressure (60 bar) at $180{ }^{\circ} \mathrm{C}$ for $5 \mathrm{~min}$ to ensure that nanotrenches in the template were fully immersed in the PFO layer; the templates were then cooled down to $135{ }^{\circ} \mathrm{C}$, held at this temperature for $10 \mathrm{~min}$, and then slowly cooled down to room temperature $\left(30^{\circ} \mathrm{C}\right)$. Before releasing the pressure, the stacks were evacuated to solidify the PFO nanogratings. The soft-NIL process is schematically shown in Fig. 1(a). The template used for this process was PDMS soft template considering its fabrication cost. The area of the PDMS stamp was about $64 \mathrm{~mm}^{2}$ and height was about $100 \mathrm{~nm}$ with $300 \mathrm{~nm}$ line-width and duty cycle around 0.5, as shown in Fig. sp1. $\dagger$ As shown in the AFM image in Fig. 1(d), the $300 \mathrm{~nm}$ line-width PFO nanogratings with different initial layer thicknesses were reproduced on the silicon wafer. By carefully tuning the initial PFO layer thickness, different filling quantities in the same PDMS stamp could be easily realized during the soft NIL process. Hence, a series of different residual and protrusion layer thicknesses could be achieved after the NIL process. A series of the protrusion layer and residual layer thicknesses are shown in Table 1, characterized by SEM shown in Fig. sp2. $\dagger$ Since the nano-trenches on the PDMS template were about
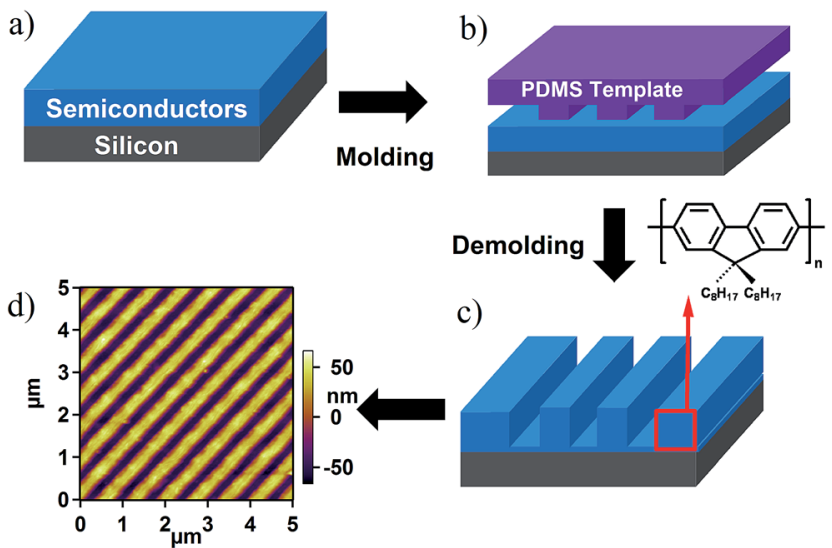

Fig. 1 One-step fabrication of the PFO nanograting. (a-c) Nanoimprinting process of the PFO nanograting. (d) AFM image of the PFO nanograting.
Table 1 The PFO nanograting configuration parameters under different initial film thicknesses and same nanoimprinting parameter

\begin{tabular}{lll}
\hline $\begin{array}{l}\text { Initial film } \\
\text { thickness }\end{array}$ & $\begin{array}{l}\text { Residual layer } \\
\text { thickness }\left(h_{\mathrm{r}}\right)\end{array}$ & $\begin{array}{l}\text { Protrusion layer } \\
\text { thickness }\left(h_{\mathrm{p}}\right)\end{array}$ \\
\hline $25 \mathrm{~nm}$ & $<10 \mathrm{~nm}$ & $40 \mathrm{~nm}$ \\
$30 \mathrm{~nm}$ & $<10 \mathrm{~nm}$ & $50 \mathrm{~nm}$ \\
$50 \mathrm{~nm}$ & $<10 \mathrm{~nm}$ & $90 \mathrm{~nm}$ \\
$65 \mathrm{~nm}$ & $\approx 10 \mathrm{~nm}$ & $90 \mathrm{~nm}$ \\
$75 \mathrm{~nm}$ & $28 \mathrm{~nm}$ & $90 \mathrm{~nm}$ \\
$90 \mathrm{~nm}$ & $49 \mathrm{~nm}$ & $90 \mathrm{~nm}$
\end{tabular}

$100 \mathrm{~nm}$ deep, the protrusions on the PFO nanograting could not exceed $100 \mathrm{~nm}$ in height, as shown in the AFM images in Fig. sp3.†

As the AFM and SEM analysis is limited to visualizing the surface morphology, GIWAXD measurement was used to analyse the orientation of the $\alpha$-conformation PFO gratings, whose incident azimuth angle was fixed at about $0.18^{\circ}$ to probe the orientation. Taking the nanogratings with $60 \mathrm{~nm}$-thickness as an example, we defined the diffraction vectors $q_{x y}$ and $q_{z}$ pointing along and normal to the substrate plane, respectively, and the lattice parameters of PFO in the orthorhombic unit were $a=2.56 \mathrm{~nm}, b=2.38 \mathrm{~nm}, c=3.32 \mathrm{~nm}^{26,27}$ The GIWAXD patterns are shown in Fig. 2(a) and (b); the incident X-ray beam is perpendicular or parallel to the long axis of nanogratings. In the $q_{z}$ direction, whether the incident X-ray beam is perpendicular or parallel to the nanogratings, the peaks at $q_{z}=4.91$ $\mathrm{nm}^{-1}$ corresponding to the (200) diffraction planes indicate the edge-on chain orientation with respect to the substrate surface. On the other hand, in the $q_{x y}$ direction, the peaks at $q_{x y}=15.14$ $\mathrm{nm}^{-1}$ corresponding to the (008) diffraction planes with the incident X-ray beam perpendicular to the nanogratings and the peaks at $q_{x y}=10.74 \mathrm{~nm}^{-1}$ and $16.11 \mathrm{~nm}^{-1}$ corresponding to the (040) and (060) diffraction planes with the incident X-ray beam parallel to the nanogratings indicate that the polymer backbone is aligned along the long axis of nanogratings.
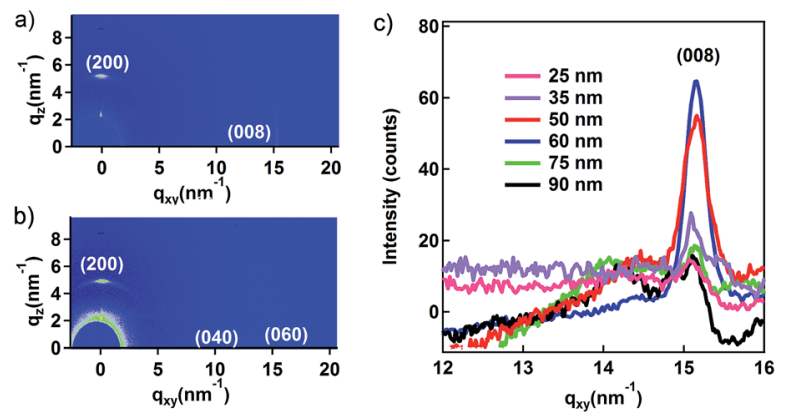

Fig. 2 Grazing incidence wide-angle X-rays diffraction (GIWAXD) of the PFO nanogratings. (a) The two-dimensional image of the PFO nanogratings with the initial film thickness about $60 \mathrm{~nm}$ and incident $X$-ray perpendicular to the nanograting. (b) The two-dimensional image with incident $X$-ray parallel to the nanograting. (c) The onedimensional integration of the two-dimensional image along $q_{x y}$ with initial film thickness in the range from $25 \mathrm{~nm}$ to $90 \mathrm{~nm}$. 
The $\alpha$-conformation PFO can be considered as a rod-like chain, which prefers to align parallel to the grooves as the graphoepitaxial alignment for geometrical and entropic reasons; thus, herein, the chain alignment in the nanograting vector is taken into account. By carefully tuning the initial film thickness of PFO, the protrusion and the residual layer thickness can be precisely controlled, such that the residual layer thickness $h_{\mathrm{r}}$ and the protrusion layer thickness $h_{\mathrm{p}}$ can be changed. Fig. 2(c) demonstrates that the reflection intensity of $\mathrm{X}$-ray on the plane (008) is changed upon changing both the protrusion and residual layer thicknesses. Based on the GIWAXD result, the crystallinity degree inside these PFO nanogratings can be tuned using different initial film thicknesses. Moreover, we can find out that the best alignment of the molecular chains occur where PFO fully fills in the nano-trench of the PDMS stamp with the lowest residual layer thickness.

To quantitatively describe the effect of the orientation degree on the initial film thickness of these embossed PFO nanogratings, the polarized UV absorption spectrum was obtained, as shown in Fig. 3. During the polarized absorption spectrum measurement, the polarizer was positioned between the sample and the incident light source. Therefore, when the sample was rotated in plane, the relative angle between the polarizer and the grating vector could be tuned manually. The starting point was set when the polarized direction was parallel to the grating vector, and the absorption spectrum is shown as a black curve in Fig. 3(a). When the relative angle was tuned to $90^{\circ}$, the absorption spectrum was obtained as a red curve, as shown in Fig. 3(a). The PFO nanogratings exhibit the maximum absorption at $390 \mathrm{~nm}$. In addition, the absorbance with the polarization parallel to the nanogratings $\left(I_{\|}\right)$is much stronger than that obtained under perpendicular polarization $\left(I_{\perp}\right)$. Since the dipole vector for light absorption of conjugated polymers is typically parallel to the backbones, the anisotropic UV-vis absorption of PFO nanogratings confirms that the polymer backbones are aligned essentially parallel to the nanogratings. ${ }^{28}$ These results are in good agreement with the structural information obtained from the GIWAXD images. The orientation degree of the embossed PFO grating with different initial film thicknesses could be calculated with the function ${ }^{29}$

$$
R=\frac{I_{\|}-I_{\perp}}{I_{\|}+2 \times I_{\perp}}
$$
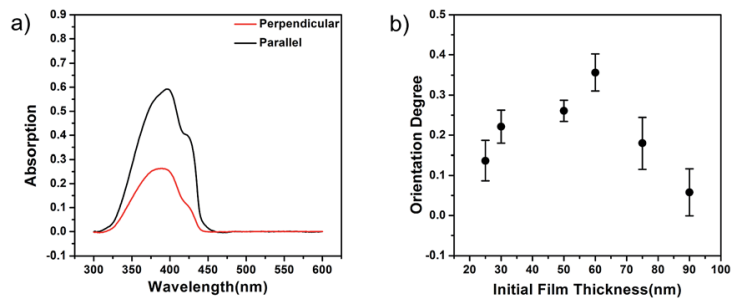

Fig. 3 Absorption analysis of the PFO nanograting. (a) The polarized absorption spectra in the inset figure with the polarized filter direction along (black line) and perpendicular to (red line) the grating vector. (b) The orientation degree with different initial film thicknesses.
(I\| $I_{\|}$corresponds to the intensity of the UV-absorption spectrum when the incident light polarized orientation is parallel to the grating vector, whereas $I_{\perp}$ is the intensity of the UV-absorption spectrum when the incident light polarized orientation is perpendicular to the grating vector). Moreover, the orientation degree of different film thicknesses shown in Fig. 3(b) indicated that the orientation degree strongly depended on the film thickness, and the highest orientation degree occurred at the initial film thickness of around $60 \mathrm{~nm}$ when PFO fully filled the nanotrench of the PDMS stamp with lowest residual layer thickness.

As shown in Fig. sp1, $\dagger$ the depth of the nanotrench was around $100 \mathrm{~nm}$. According to the volume conversation law, the thickness of the initial layer should be equal to half of the protrusion and residual thickness. Hence, since the initial layer thickness is lower than $50 \mathrm{~nm}$, the nano-trenches cannot be fully filled by PFO, as shown in Fig. sp $3, \dagger$ and the initial layer thickness higher than $50 \mathrm{~nm}$ can result in fully filled nanotrenches and tuneable residual layer thickness. The protrusion thickness was fixed once the initial layer thickness became higher than $50 \mathrm{~nm}$, as shown in the AFM images in Fig. sp3(b) and (c). $\dagger$ As mentioned in ref. 12, confinement scaling can result in different molecular chain alignments; thus, herein, we have discussed the molecular chain alignment mechanism inside the nanogratings after the soft-NIL process. As shown in Fig. sp $2, \uparrow$ the imprinting depth and the residual layer thickness could be carefully tuned by different filling states. Before PFO at the initial quantity could fully fill the PDMS stamp, the residual layer thickness was kept constant at around $10 \mathrm{~nm}$. Moreover, the protrusion depth inside the PDMS nano-trenches could be tuned by the initial film thickness. As the protrusion depth increased, the molecular chain arrangement on the top surface was affected by undersurface of the PDMS stamp due to the graphoepitaxial alignment. For geometrical and entropic reasons, the molecular chain orientation was chosen to follow the attachment surface. At the initial film thickness of around 25 or $30 \mathrm{~nm}$, only the side surfaces could provide attachment surfaces for the graphoepitaxial alignment. As the initial film thickness increased, the filling quantity increased as well. Hence, the undersurface in PDMS nanotrenches also became the attachment surface. The top surfaces of the PFO nanogratings were not free any more. All the three surfaces inside the nanotrenches were attachment surfaces for the graphoepitaxial alignment. The nanotrenches in PDMS could be considered as a one-dimensional channel with infinite length. Therefore, the molecular chains prefer to be arranged along the nanotrenches to reduce the volume entropy. When PFO fully filled the nanotrenches, the molecular chain alignment inside the protrusion part reached the upper limit. As the initial film thickness continued to increase, the molecular chain alignment in the protrusion part could not improve further. However, the residual layer was thickened. In the residual layer, the molecular chains were freely arranged without any preferred orientation in the substrate plane as a matter of the entropy principle. Thus, as the residual layer thickened, the overall average of order degree of the (008) planes in the entire PFO nanograting was declined, as shown in Fig. 2. 
Besides the X-ray diffraction analysis, polarized absorption was measured to verify this deduction. The PFO nanograting with the initial film thickness of around $60 \mathrm{~nm}$ embodied the best performance in the orientation degree. As the $60 \mathrm{~nm}$ initial film thickness sample underwent the fully filled state during the nanoimprint process, the (008) planes uniformity reached the upper limit. On the other hand, the residual layer was too thin to affect the entire arrangement of the (008) planes. Hence, the PFO nanograting with the initial film thickness of around $60 \mathrm{~nm}$ possessed the best performance in the (008) plane uniformity and the orientation degree. As previously reported, the transition dipoles were off-axis in the molecular chain direction and made an angle of about $26^{\circ} .^{30}$ Therefore, the absolute values of the orientation degree and the polarized rate were not very high. We were convinced that the involvement of the template undersurface could improve the molecular chain alignment further in the soft-NIL process. By the precise control of the filling content, the maximum thick protrusion layer and the minimum thick residual layer could be realized and result in the best molecular chain alignment; this could be greatly helpful in improving the optoelectronic devices.

Since the filling degree in nanotrenches of the PDMS templates had a strong influence on the crystallinity degree and orientation degree, the luminescence property should be optimized by optical pumping. Hence, the polarizability could be tuned; thus, the polarized photoluminescence spectrum was obtained, as shown in Fig. 4(a and b). Polarized photoluminescence spectra were also used to investigate the optical pumping properties of the embossed PFO nanogratings. The polarized photoluminescence spectrum is shown in Fig. 4(b) with the analyzer set between the sample and the emitting detector. The parallel component (the black curve) and the perpendicular component (the red curve) of the photoluminescence spectrum with the initial film thickness of about $60 \mathrm{~nm}$ are shown in Fig. 4(a). The polarized rate was an
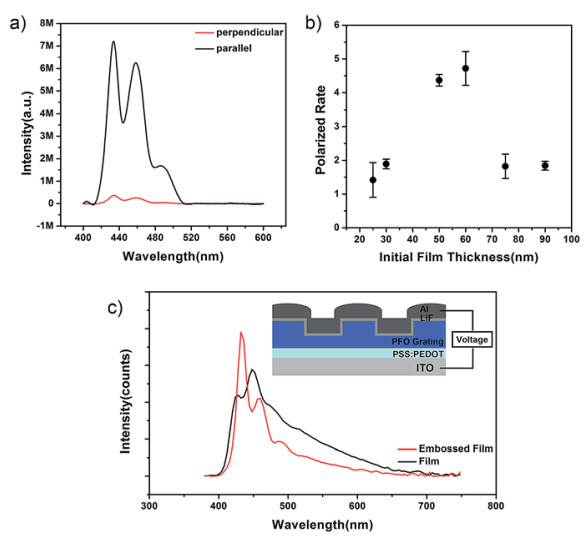

Fig. 4 The emission characterization by optical and electrical pumping. (a) The polarization analysis of the emitting light from PFO gratings with the analyzer filter direction along (black line) and perpendicular to (red line) the grating vector. (b) The polarized rate with different initial film thicknesses. (c) The light-emitting intensities at the applied voltage of $5 \mathrm{~V}$ on the PFO films with the initial thickness of about $60 \mathrm{~nm}$. important factor to study the polarized-emitting behaviours and calculated using the function ${ }^{29}$

$$
P=\frac{I_{\|}}{I_{\perp}}
$$

(where $I_{\|}$is the intensity of the analyzer parallel to the grating vector, whereas $I_{\perp}$ is the intensity of the analyzer perpendicular to the grating vector). As shown in Fig. 4(b), the polarizability of the emission light increased with an increase in protrusion thickness and decreased with an increase in the residual layer thickness. Moreover, the maximum polarized rate was obtained at the initial film thickness of around $60 \mathrm{~nm}$ when PFO fully filled the nano-trench of the PDMS stamp with lowest residual layer thickness.

Compared to the surface assistant molecular alignment, this nanoconfinement system in nanoimprint lithography can simultaneously induce the surface structure and the molecular chain alignment. The traditional 5-layer structured PLED was used to study the electronic light-emitting properties of PFO gratings with inner molecular alignment. In this part, about $20 \mathrm{~nm}$ PSS:PEDOT film was spin-coated on the patterned ITO electrode; then, $\alpha$-conformation PFO nanogratings were fabricated on this $20 \mathrm{~nm}$ PSS:PEDOT film by nanoimprinting. After this, about $1 \mathrm{~nm} \mathrm{LiF}$ and $100 \mathrm{~nm} \mathrm{Al}$ were evaporated on the $\alpha$ conformation PFO nanogratings. The schematic of the PLED is shown in Fig. 4(c). The electroluminescence (EL) spectrum shown in Fig. 4(c) at the applied voltage of about $5 \mathrm{~V}$ indicates that the PFO nanogratings can extract light $20 \%$ more than the flat unprocessed films. Herein, samples with the film thickness of about $60 \mathrm{~nm}$ are chosen. The embossed PFO nanograting and unembossed PFO film were studied. Although the amount of the luminescent polymer PFO is equal in both cases, the EL intensity of PFO nanogratings with embossed surface is much higher. Many efforts have been focused on luminescence enhancement by inducing patterns of the devices. ${ }^{31-35}$ Among them, luminescence enhancement can be induced in two ways: (i) modulating the period of the pattern: if the period is about half of the emitting wavelength, the luminescence is enhanced by the output coupling and scattering effect; ${ }^{31}$ (ii) inducing metal, such as Ag, nanostructures into the pattern; these nanostructures enhance the luminescence by the weak surface plasmon and scattering effects. ${ }^{32}$ However, in our studies, the period of the PFO gratings is about $600 \mathrm{~nm}$, which is much higher than the half period of the emitting wavelength (450 $\mathrm{nm}$ ); this results in almost no output coupling and scattering effect. In addition, the height of the PFO nanogratings is lower than $90 \mathrm{~nm}$, whereas the thickness of the Al electrode in our experiments is about $100 \mathrm{~nm}$; hence, the surface plasmonic effect becomes very weak. To study the reason for luminescence enhancement of PFO nanogratings, we measured the photoluminescence quantum efficiency (PQE) of the PFO nanogratings and unprocessed thin films. The PQE is about $36 \%$ for the PFO nanogratings, which is about $25 \%$ higher than that of the unprocessed thin films (the PQE is about 28\%); the PQE improvement can be attributed to the molecular and crystalline alignment, and this improvement results in the PFO 
nanogratings being able to extract $20 \%$ more light than the flat unprocessed films.

\section{Experimental}

\section{Preparation of the $\alpha$-conformation PFO film}

The PFO (poly(9,9-dioctylfluorene)) (ADS129BE) was purchased from American dye source company and used as received. The PFO powder was dissolved in toluene and heated to $60{ }^{\circ} \mathrm{C}$ overnight for sufficient dissolution. The solution was filtered using $0.25 \mu \mathrm{m}$ polytetrafluoroethylene filters. The PFO thin films were prepared by spin-coating. The substrates used were quartz glass and the silicon wafer, cleaned using a freshly prepared piranha solution $\left(\mathrm{H}_{2} \mathrm{O}_{2}(27 \%)\right.$ and $\left.\mathrm{H}_{2} \mathrm{SO}_{4}(98 \%) 1: 3\right)$. The film thickness depended on both the spin speed and the solution concentration. To control the film thickness in the range between $25 \mathrm{~nm}$ and $90 \mathrm{~nm}$, the solution concentration was chosen in the range between $10 \mathrm{mg} \mathrm{ml}^{-1}$ and $20 \mathrm{mg} \mathrm{ml}^{-1}$, and the spin speeds were between $2000 \mathrm{rpm}$ and $4000 \mathrm{rpm}$. The $\alpha$-conformation thin film achieved by thermal annealing at $180^{\circ} \mathrm{C}$ for 5 minutes was slowly cooled down to $135^{\circ} \mathrm{C}$ and then slowly cooled down to the room temperature.

\section{Fabrication of the PFO nanogratings}

The thin films were transferred to a nano-imprinter (Obducat, Eitre 3) and covered with PDMS (polydimethylsiloxane) template replicated from the polymethylmethacrylate (PMMA) template by E-beam lithography. The height of the PDMS template is about $100 \mathrm{~nm}$. The PDMS templates were pressed against the films under pressure (60 bar) at $180^{\circ} \mathrm{C}$, held at this temperature for $5 \mathrm{~min}$ to ensure that nano-trenches in the template were completely filled, then cooled down to $135{ }^{\circ} \mathrm{C}$, held at this temperature for $10 \mathrm{~min}$, and then slowly cooled down to room temperature $\left(30^{\circ} \mathrm{C}\right)$. Before releasing the pressure, the stacks were evacuated to solidify the PFO nanogratings.

\section{Fabrication of polymer light-emitting diodes (PLEDs)}

The traditional 5-layer structure PLED was used in this study to check the performance improvement by tuning the molecular chain alignment inside. In this part, about $20 \mathrm{~nm}$ PSS:PEDOT film was spin-coated on the patterned ITO electrode. Then, $\alpha$ conformation PFO nanograting was fabricated on this $20 \mathrm{~nm}$ PSS:PEDOT film by a nano-imprinter. Moreover, about $1 \mathrm{~nm} \mathrm{LiF}$ and $100 \mathrm{~nm} \mathrm{Al}$ were evaporated from the $\alpha$-conformation PFO nanogratings by high vacuum thermal evaporation with a shadow mask, the area of which was about $9 \mathrm{~mm}^{2}$, which was smaller than that of the PFO nanogratings $\left(64 \mathrm{~mm}^{2}\right)$.

\section{Characterization}

The thickness of the homogeneous films was measured by a film thickness measurement instrument (F20, Filmetrics, USA) with 1.49 as the refractive index. The morphologies of the PFO films were characterized in the tapping mode of the atomic force microscope (AFM, MFP-3D-SA, Asylum Research, USA) using Pt-coated Si cantilevers $\left(0.2 \mathrm{~N} \mathrm{~m}^{-1}\right.$ force constant from
Nanosensors) and a scanning electronic microscope (SEM, Pioneer, Raith, Germany) at $10 \mathrm{kV}$. Grazing incidence wideangle X-rays diffraction (GIWAXD) measurements were carried out at the BL14B1 beam line at the Shanghai synchrotron radiation facility (SSRF) in China. The wavelength and the incident angle of the X-ray beam are $0.12398 \mathrm{~nm}$ and $0.18^{\circ}$, respectively. The distance between the sample centre and the two-dimensional charge-coupled device (CCD) detector is 315 $\mathrm{mm}$. Data conversion to $q$ space was accomplished by calibration using $\mathrm{LaB}_{6}$ powder. Polarized UV absorption spectra (UV3600, Shimadzu, Japan) was used to study the orientation degree of PFO nanogratings with different film thicknesses in the transmission mode using a Glan-Thomson polarizer positioned between the sample and the incident light source. The polarized PL spectra (spectrofluorometer, Fluoromax-4, Horiba, USA) were used to study the polarized emission behaviours of the patterned $\alpha$-conformation PFO film with $380 \mathrm{~nm}$ excitation wavelength, and the analyzer was positioned ahead of the emitting detector. The current-voltage and the luminance spectrum characteristics were measured using a constant current source (SourceMeter, Keithley 2400, USA) combined with a photometer (SpectraScan, PR 655, Photo Research, USA). The PQE of the PFO gratings and films were measured using an FLS980 spectrometer (Edinburgh Instruments).

\section{Conclusions}

A one-step method has been developed to fabricate a PFO thin layer, which can achieve three functions at the same time: patterning, molecular chain alignment, and fluorescence enhancement. Based on the lithography process, patterning and molecular chain alignment can be achieved. Additionally, the crystallinity and the orientation degree can be tuned only by adjusting the PFO layer thickness before the nanoimprinting process, called the initial film thickness. When the initial film thickness reaches the critical value, it makes nanoconfinement the most and the residual layer the least, and this PFO nanograting can reflect highest crystallinity and orientation, as shown in Fig. 2 and 3. We have built the 5-layer PLED devices, and the EL intensity of the PFO nanogratings is $20 \%$ higher than that of the flat unprocessed films. We anticipate that the method described herein can also be applied to a wider preparation of materials for the production of high-performance PLEDs due to the ease of use and a substantial increase in device performance.

\section{Conflicts of interest}

There are no conflicts to declare.

\section{Acknowledgements}

This work was financially supported by the National Nature Science Foundation of China (No. 21204058, 21074084, 21374069, 21104051 and 91027040) and the BL14B1 Beamline at the Shanghai Synchrotron Radiation Facility in China. 


\section{Notes and references}

1 P. D. Cunningham, J. B. Souza Jr, I. Fedin, C. X. She, B. Lee and D. V. Talapin, ACS Nano, 2016, 10(6), 5769.

2 S. I. Jo, Y. Kim, J. H. Baek, C. J. Yu and J. H. Kim, Jpn. J. Appl. Phys., 2014, 53, 03 CD04.

3 A. Dauendorffer, S. Miyajima, S. Nagamatsu, W. Takashima, S. Hayase and K. Kaneto, Appl. Phys. Express, 2012, 5, 09210.

4 M. F. Toney, T. P. Russell, J. A. Logan, H. Kikuchi, J. M. Sands and S. K. Kumar, Nature, 1995, 374, 709.

5 D. Kawakami, B. S. Hsiao, C. Burger, S. F. Ran, C. Avila-Orta, I. Sics, T. Kikutani, K. I. Jacob and B. Chu, Macromolecules, 2005, 38, 91.

6 K. Jradi, S. Bistac, M. Schmitt and G. Reiter, Polymer, 2009, 50, 3724 .

7 N. Tanigaki, C. Heck and T. Mizokuro, Mol. Cryst. Liq. Cryst., 2009, 505, 318.

8 T. Mizokuro, Y. Okamoto, C. Heck, H. Aota and N. Tanigaki, J. Appl. Polym. Sci., 2014, 131, 631.

9 S. D. Siqing, H. Wu and A. Takahara, Polymer, 2015, 72, 113. 10 T. Matsui, K. Nakayama, M. Ozaki and K. Yoshino, Appl. Phys. Lett., 2000, 76, 1228.

11 W. Hu, A. K. Srivastava, X. W. Lin, X. Liang, Z. J. Wu, J. T. Sun, G. Zhu, V. Chigrinov and Y. Q. Lu, Appl. Phys. Lett., 2012, 100, 111116.

12 Z. J. Hu and A. M. Jonas, Soft Matter, 2010, 6, 21.

13 Z. J. Hu, B. Muls, L. Gence, D. A. Serban, J. Hofkens, S. Melinte, B. Nysten, S. Demoustier-Champagne and A. M. Jonas, Nano Lett., 2007, 7, 3639.

14 Z. J. Hu, M. W. Tian, B. Nysten and A. M. Jonas, Nat. Mater., 2009, 8, 62.

15 Q. Zhang, L. Chi, G. Hai, Y. T. Fang, X. C. Li, R. D. Xia, W. Huang and E. Gu, Molecules, 2017, 22, 315.

16 D. Abbaszadeh, N. Y. Doumon, G. A. H. Wetzelaer, L. J. A. Koster and P. W. M. Blom, Synth. Met., 2016, 215, 64. 17 A. P. Kulkarni and S. A. Jenekhe, Macromolecules, 2003, 36, 5285.

18 K. Sakamoto, K. Miki, M. Misaki, K. Sakaguchi, M. Chikamatsu and R. Azumi, Appl. Phys. Lett., 2007, 91, 183509.
19 K. Sakamoto, K. Miki, M. Misaki, K. Sakaguchi, Y. Hijikata, M. Chikamatsu and R. Azumi, J. Appl. Phys., 2010, 107, 113108.

20 L. Ghirardini, T. Virgili, S. Bolis, J. Beeckman, P. Kockaert, M. Finazzi and M. Celebrano, J. Polym. Sci., Part B: Polym. Phys., 2016, 54, 1558.

21 J. Martin, A. D. Scaccabarozzi, A. Nogales, R. P. Li, D. M. Smilgies and N. Stingelin, Eur. Polym. J., 2016, 81, 650.

22 X. H. Li, J. C. Yu, N. Y. Lu, W. D. Zhang, Y. Y. Weng and Z. Gu, Chin. Phys. B, 2015, 24, 104215.

23 R. K. Sharma and M. Katiyar, Org. Electron., 2016, 38, 121.

24 M. Kitamura, S. Iwamoto and Y. Arakawa, Jpn. J. Appl. Phys., 2005, 44, 2844.

25 Y. S. Shim, K. N. Kim, J. H. Hwang, C. H. Park, S. G. J. Y. W. Park and B. K. Ju, Nanotechnology, 2016, 28, 045301.

26 S. H. Chen, H. L. Chou, A. C. Su and S. A. Chen, Macromolecules, 2004, 37, 6833.

27 S. H. Chen, A. C. Su, C. H. Su and S. A. Chen, Macromolecules, 2005, 38, 379.

28 M. C. Gurau, D. M. DeLongchamp, B. M. Vogel, E. K. Lin, D. A. Fischer, S. Sambasivan and L. J. Richter, Langmuir, 2007, 23, 834.

29 Z. J. Zheng, K. H. Yim, M. S. M. Saifullah, M. E. Welland, R. H. Friend, J. S. Kim and W. T. S. Huck, Nano Lett., 2007, 7, 987.

$30 \mathrm{H}$. M. Liem, P. Etchegoin, K. S. Whitehead and D. D. C. Bradley, Adv. Funct. Mater., 2003, 13, 66.

31 L. Zhou, X. C. Jiang, Y. Q. Li, A. L. Shi, J. D. Chen, Q. D. Ou, H. T. Liu and J. X. Tang, ACS Appl. Mater. Interfaces, 2014, 6, 18139.

32 Y. F. Liu, M. H. An, X. L. Zhang, Y. G. Bi, D. Yin, Y. F. Zhang, J. Feng and H. B. Sun, Appl. Phys. Lett., 2016, 109, 193301.

33 C. Lee, K. H. Han, K. H. Kim and J. J. Kim, Opt. Express, 2016, 21, A489.

34 K. J. Byeon, H. Park, J. Y. Cho, S. H. Lee, S. Y. Lee, J. O. Song and H. Lee, Current Applied Physics, 2011, 11, S147.

35 T. B. Lim, K. H. Kim, S. A. Song, S. N. Lim, K. Y. Kim and Y. C. Jeong, Macromol. Res., 2017, 25, 786. 